Journal Pre-proof

GraPASA: Parametric Graph Embedding via Siamese Architecture

Yujun Chen, Ke Sun, Juhua Pu, Zhang Xiong, Xiangliang Zhang

PII: S0020-0255(19)30980-6

DOI: https://doi.org/10.1016/j.ins.2019.10.027

Reference: INS 14943

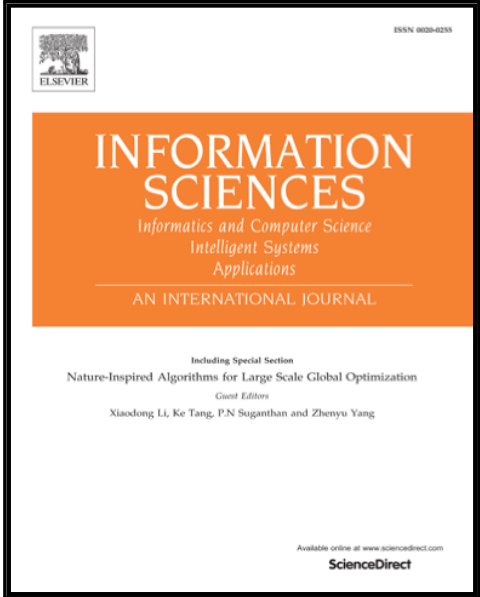

To appear in:

Information Sciences

Received date: $\quad 3$ July 2018

Revised date: $\quad 6$ August 2019

Accepted date: $\quad 13$ October 2019

Please cite this article as: Yujun Chen, Ke Sun, Juhua Pu, Zhang Xiong, Xiangliang Zhang, GraPASA: Parametric Graph Embedding via Siamese Architecture, Information Sciences (2019), doi: https://doi.org/10.1016/j.ins.2019.10.027

This is a PDF file of an article that has undergone enhancements after acceptance, such as the addition of a cover page and metadata, and formatting for readability, but it is not yet the definitive version of record. This version will undergo additional copyediting, typesetting and review before it is published in its final form, but we are providing this version to give early visibility of the article. Please note that, during the production process, errors may be discovered which could affect the content, and all legal disclaimers that apply to the journal pertain.

C) 2019 Published by Elsevier Inc. 


\title{
GraPASA: Parametric Graph Embedding via Siamese Architecture
}

\author{
Yujun $\mathrm{Chen}^{\mathrm{a}}$, Ke Sun ${ }^{\mathrm{c}}$, Juhua $\mathrm{Pu}^{\mathrm{a}}$, Zhang Xiong ${ }^{\mathrm{a}}$, Xiangliang Zhang ${ }^{\mathrm{b}, *}$ \\ ${ }^{a}$ School of Computer Science and Engineering, Beihang University, \\ No.37 Xueyuan Road, 100191, Beijing, China \\ ${ }^{b}$ Division of Computer, Electrical and Mathematical Sciences 83 Engineering, \\ King Abdullah University of Science and Technology (KAUST), Saudi Arabia \\ ${ }^{c}$ CSIRO's Data61, Australia
}

\begin{abstract}
Graph representation learning or graph embedding is a classical topic in data mining. Current embedding methods are mostly non-parametric, where all the embedding points are unconstrained free points in the target space. These approaches suffer from limited scalability and an over-flexible representation. In this paper, we propose a parametric graph embedding by fusing graph topology information and node content information. The embedding points are obtained through a highly flexible non-linear transformation from node content features to the target space. This transformation is learned using the contrastive loss function of the siamese network to preserve node adjacency in the input graph. On several benchmark network datasets, the proposed GraPASA method shows a significant margin over state-of-the-art techniques on benchmark graph representation tasks.
\end{abstract}

Keywords: Network embedding; inductive representation learning; Siamese network; information fusion

\footnotetext{
${ }^{*}$ Corresponding author

Email address: chenjohn@buaa.edu.cn (Yujun Chen), sunk.edu@gmail.com (Ke Sun), pujh@buaa.edu.cn (Juhua Pu), xiongz@buaa.edu.cn (Zhang Xiong), xiangliang.zhang@kaust.edu.sa (Xiangliang Zhang),
} 


\section{Introduction}

With the fast development of graph representation learning tasks in recent years, many network related tasks obtain better results from low-rank graph representation methods. A variety of graph representation methods show their

5 effectiveness in dealing with graph related tasks like, node classification, link prediction and graph reconstruction $[10,14,16]$. Many graph representation methods recently are inspired from neural language models [3, 32]. Some methods learned the graph representations from network topology [39, 42, 19, 12, 41], while others incorporate node content information for learning the graph repre10 sentation $[49,50,20,1,11,31]$. In general, recent graph representation methods utilize dimensionality reduction techniques to distill the high-dimensional information about nodes' neighborhood into the distributed representation vector. The representation can then be fed to other machine learning systems and boost various graph mining tasks.

15 The current development on graph embeddings is largely based on the nonparametric approach, where each graph node is embedded as a free point in $\Re^{d}$ ( $d$ : dimension of the target space) [27]. Therefore in total we have $O(n d)$ free parameters to learn, where $n$ is the number of nodes. This approach faces a huge number of free parameters as the network scales up. Moreover, it does not provide an out-of-sample mapping from the graph nodes to the target space. Hence the learned representation model cannot generalize to unobserved nodes at training time. The issue is partially addressed by re-learning the node representation incrementally $[39,49]$ at the cost of a high computational overhead.

Parametric graph embedding approaches are under development in another thread. In this thread, embeddings are defined as a parametric function of the feature vectors. Nickel and Jiang et.al. [36] showed that linear embeddings of graph requires large dimensionality to capture complex relations, while nonlinear embedding can mitigate this problem [5, 4]. Sophisticated non-linear mappings represented by a recurrent neural network have been applied to the graph domain [18]. In a semi-supervised setting, Yang et.al. [50] and Hamilton 
et.al. [20] introduced a parametric mapping to embed the node features. The Graph convolution networks [23] also provide a parametric mapping based on graph spectral theory.

This paper follows the latter parametric approach. In this paper, we learn a 35 non-linear mapping parametrized by a deep neural network to fuse node content information with node adjacency information in a clever way: the input of the mapping is the raw node contents. After the mapping, the output is constrained to respect node adjacency based on the Siamese cost function [6]. Both node pairs with similar contents and node pairs with short graph distances will be embedded nearby, according to the graph homophily theory [22]. These two kinds of information are thus tightly integrated by a unified cost function. Also, to obtain embedding without task-specific supervision, we focus on learning the graph embeddings in an unsupervised manner. The proposed method is named GraPASA for Graph PArametric representation with Siamese Architecture. It stands out from alternative models on the following aspects: (1) GraPASA is a parametric method and can be easily applied to both transductive and inductive network learning tasks. (2) GraPASA elegantly integrates network topology and node content information based on the contrastive loss. (3) GraPASA is a purely unsupervised approach and is therefore useful to general application purposes.

We compare the proposed method with several state-of-the-art unsupervised graph embedding methods including Node2Vec [19], TADW [49] PLANETOID [50], GraphSAGE [20], Graph2Gauss [4], and pRBM [48] on node classification, community detection and graph visualization tasks. The experimental results on three real-world networks show that GraPASA consistently outper55 forms the alternatives with a notable margin.

Our main contributions are summarized as follows:

- An efficient parametric node embedding method, GraPASA, for both inductive and transductive graph representation learning.

- A novel application of the Siamese network in the area of graph learning and a new "graph neural network". 
- Extensive empirical study on comparing the proposed method and several state-of-the-art graph embedding methods on different graph mining tasks.

The rest of this paper is organized as follows. Section 2 discusses related works in graph representation learning and parametric embedding learning. Sec-

65 tion 3 presents the proposed GraPASA model. Section 4 reports our experiments results. Section 5 concludes and hints at future perspectives.

\section{Related Works}

\subsection{Graph representation Learning}

A great amount of attention has been paid to representation learning problems in graphs/networks, which is also called graph/network embedding. The related work can be categorized as unsupervised or semi-supervised learning approaches depending upon the usage of given labels in the learning process. Related works can also be divided by checking if only the graph topology information is used for learning, or other information is additionally employed, e.g., 75 node content information. The study in unsupervised representation learning with only the topology information has a big family of developed approaches. The network topology is usually represented by an adjacency matrix, $A_{(|V| *|V|)}$, where $A_{i j}$ is 1 if node $v_{i}$ and $v_{j}$ are connected, or is a numeric weight value if the edge connecting $v_{i}$ and $v_{j}$ has a weight, otherwise $A_{i j}$ is 0 . To obtain

80 node representation in $\Re^{d}$, dimensionality reduction techniques like singular value decomposition (SVD) or principal component analysis (PCA), and multidimensional scaling (MDS) can be applied on $A$ or graph Laplacian matrix and Modularity matrix derived from $A$ [43]. However, the poor scalability of these approaches makes them difficult to be applied to large-scale networks.

Recently, another stream of work addresses the unsupervised representation learning of nodes in large-scale graph with an inspiration from neural language models (e.g., word2vec in [32]). Deepwalk [39] and node2vec [19] learn node representation that maximizes the co-occurrence probability of a target node and its context nodes, which are generated by short random walks in networks. 
Based on the similar notion of "context", LINE [42] is proposed to explicitly preserve the first-order and second-order proximity between nodes. Following the proof that the Skip-Gram model in word2vec with negative sampling [32] is implicitly factorizing a word-context matrix in [24], Yang et al. show that Deepwalk is equivalent to matrix factorization also when the factorized matrix 95 is designed to show the probability that a node reaches another by random walks [49].

In this set of semi-supervised representation learning work, available labels guide the learned representation for better predictive capacity. After the presence of Deepwalk, Yang et al. propose a transductive and inductive framework (Planetoid) for learning the representation for each graph node to jointly predict the class label and the neighborhood context in the graph [50]. A method called max-margin DeepWalk (MMDW) proposed in [45] jointly optimizes the maxmargin classifier and the representation learning model formulated in a matrix factorization manner. Similarly, Discriminative Deep Random Walk (DDRW) proposed in [25] jointly learns a classifier and vertex representation by combining the loss function of SVM and that of Skip-gram model.

All the above-mentioned approaches learn non-parametric representation for graph nodes, which differ from the parametric mapping learned by GraPASA in this paper. The inductive model of Planetoid [50] defines a node representation as a parameterized function of node content feature. However, unlike the these methods, the proposed GraPASA in this paper is an unsupervised model and follows a totally different problem formulation.

GraphSAGE [20] and SDNE [46] are also designed for inductive network learning. But both GraphSAGE and SDNE calls for topology feature to infer the representation vector, which is not scalable to sparsely connected graph. Graph2Gauss [4] regarding embeddings as distributions can also be used for inductive network learning, but Graph2Gauss cannot capture complex non-linear mapping because it is a shallow network model. Also, the variance of distributional embedding will impact the node classification performance. GraPASA can infer network embedding only by content features, and GraPASA is a deep 
network model, which can fit more complicated non-linear mapping.

Also many of these unsupervised and semi-supervised learning approaches solve representation learning problem from only the topology information, except $[49,37,51]$, which incorporated with node content information and other available graph information. However, methods like TADW [49] and HSCA [51] require matrix operation like SVD decomposition, which prohibits them from dealing with large scale graphs. TriDNR in [37] is a semi-supervised method and requires extra label information.

\subsection{Deep Siamese Network}

Deep learning has been recognized as a promising solution to problems across several research fields [17]. Deep learning also played in network embedding. Auto-encoder is used in [47] with first-order and second-order proximity for designing a semi-supervised deep learning model for network embedding. Autoencoder is also employed in [7] for learning a non-linear mapping from vector representations constrained by a positive point-wise mutual information (PPMI) matrix to low-dimensional vertex representations. Convolutional Neural Networks helped on learning embedding of heterogeneous network in [9]. However, these models focus mainly on learning from only the topology information.

Siamese network, another architecture of deep neural networks, was first introduced in the early 1990s by Bromley and LeCun [6] to solve signature verification as an image matching problem. The Siamese network did not achieve great attentions until the deep learning boom in the late 2000s [13]. Since the successful application in unsupervised feature learning, many more feature learning tasks begin to use the Siamese network recently [33, 34]. The Siamese Network incorporates the contrastive information between a pair of inputs to explore essential similarity or dissimilarity in the representation space. For our application, the Siamese Network can establish a representation space that embeds similar node pairs nearby as these node pairs have similar contents and short graph distances, while node pairs that differ in content and/or are distant in graphs are embedded in separated areas. The node content and node 
adjacency in graph structure are tightly integrated by a unified cost function in the Siamese Network.

\subsection{Manifold Learning}

The two areas of graph embedding and manifold learning, a.k.a. nonlinear dimensionality reduction, are well connected and share similar techniques. While graph embedding learns a mapping from the graph nodes to a lowdimensional target space $\Re^{d}$, the task of manifold learning is to learn a non-linear mapping from the observation space (usually $\Re^{D}$ ) to $\Re^{d}$

It is worth to mention that similar methodologies to preserve input adjacency information appear earlier in the literature of manifold learning (see e.g., [2, 21]). It is therefore meaningful to borrow methodologies from manifold learning into the area of graph embedding.

On the other hand, the graph embedding community has widely adopted negative sampling and stochastic gradient descent to scale to large datasets.

This could be interesting to manifold learning, where efforts are made to tackle (see e.g., [29]) the $O\left(n^{2}\right)$ complexity of batch gradient descent.

The development of manifold learning follows both a parametric approach e.g., auto-encoders and principal component analysis, as well as a non-parametric approach, e.g., Locally Linear Embedding (LLE) [40] and Laplacian Embedding [2]. There have been related efforts $[28,8]$ to port non-parametric manifold learning into a parametric way, which is similar to the proposed GraPASA. However, GraPASA is uniquely designed for graph embedding and graph feature fusion.

\section{Methodology}

In this section, we will first introduce the problem definition and notations be used. Then we will present the idea of parameterizing previous Deep Walk method. After that, we will describe how to build nodes relationship in detail via the siamese model and non-linear function in our work. 


\subsection{Problem Definition and Notations} the set of nodes, and $E \subseteq V \times V$ is the edge set including connections between nodes. $\mathbf{X}=\left\{X_{1}, X_{2}, \ldots, X_{n}\right\}$ is the set of random variables, in which $X_{i}$, taking its values in a space $\mathcal{X}$, describes features of the node $v_{i}$. For traditional non-parametric graph representation method, a specific representation will be learned for each node.

In this paper, node representation learning is to find a mapping $f_{\theta}$, which projects content feature $X_{i}$ of node $v_{i}$ in a graph as a vector $f_{\theta}\left(X_{i}\right) \in \Re^{d}$ in $d$-dimensional space, given the node adjacency and node content information in graph $G$. Here, $\theta$ is the parameter of the mapping that will be learned. $d$ is a pre-specified parameter denoting the number of dimensions of node representation. The learned representation $f_{\theta}\left(X_{i}\right)$ will be used in various tasks like node classification and network clustering.

\subsection{Parameterized Objective Function}

The recent popular methods like Deepwalk [39] and node2vec [19] formalize the graph representation learning problem as the optimization of an objective function that maximizes the log-probability of the co-occurrence in a graph neighborhood $N_{S}\left(v_{i}\right)$ for the target node $v_{i}$, given $v_{i}$ 's representation $f\left(v_{i}\right)$. For example, the objective function of node2vec is

$$
\max _{f} \sum_{v_{i} \in V} \sum_{v_{c} \in N_{S}(v)} \log \operatorname{Pr}\left(f\left(v_{c}\right) \mid f\left(v_{i}\right)\right)
$$

where for target node $v_{i} \in V, N_{S}\left(v_{i}\right) \subset V$ is defined as the neighborhood nodes (a.k.a. context) of node $v_{i}$, and is generated by a particular graph sampling strategy $S$. Notice that the node representation $f\left(v_{i}\right)$ or $f\left(v_{c}\right)$ in node2vec is not parametrized and the representation is a free embedding point in $\Re^{d}$.

In this paper, we target at learning a parametric mapping $f_{\theta}\left(X_{i}\right)$ as node representation, which is a non-linear function of node content features $X_{i}$ for node $v_{i}$, with constraints of network structure. Following the formulation in 
Eq. (1), we maximize the log-probability of the co-occurrence of neighborhood nodes integrating node feature $X$ in $N_{S}(v)$ by using parametric mapping $f_{\theta}$ :

$$
\max _{\theta} \sum_{v_{i} \in V} \sum_{v_{c} \in N_{S}\left(v_{i}\right)} \log \operatorname{Pr}\left(f_{\theta}\left(X_{c}\right) \mid f_{\theta}\left(X_{i}\right)\right)
$$

where $f_{\theta}(\cdot)$ is the parametric mapping, same for all nodes in $V$. Although Eq. (2) is formalized as a maximum likelihood optimization, it directly generalizes to any distance metric between $f_{\theta}\left(X_{c}\right)$ and $f_{\theta}\left(X_{i}\right)$. This will be discussed in detail in the following subsection. Moreover, unlike $f$ in non-parametric methods, the parametric mapping $f_{\theta}$, once learned, can be used to produce the representation $f_{\theta}(X)$ for both observed and unobserved node $v$, by simply applying $f_{\theta}$ on feature $X$.

\subsection{Sampling Strategy}

Node2vec proposed a generalized sampling strategy for obtaining $N_{S}(v)$ for each node $v$ and has shown its superiority over other methods like Deepwalk and LINE [19]. We thus follow the 2-nd order random walk algorithm in node2vec for obtaining the neighborhood nodes, and generate positive samples. Negative samples input to the Siamese network is extracted by negative sampling [32]. This is very similar to the sampling strategy proposed in Planetoid [50]. However, unlike Planetoid that uses the supervised labeling information, the sampling here is purely unsupervised.

In our case, we sample a set of triplets $\left(v_{i}, v_{c}, \delta\right)$ from networks, where $v_{i}$ and $v_{c}$ denote a target node and one of its context nodes respectively, $\delta=1$ indicates $\left(v_{i}, v_{c}\right)$ is a positive node pair, and $\delta=0$ indicates $\left(v_{i}, v_{c}\right)$ is a negative pair. A parameter $p_{1} \in(0,1)$ is set to control the ratio of sampled positive and negative pairs.

The sampling process of one triplet is listed in Algorithm 1. First, a walk sequence $S=\left\{S_{1}, S_{2}, \ldots, S_{j}, \ldots, S_{l}\right\}$ is generated by using the 2-nd order random walk algorithm in node2vec, where $S_{j}$ is the $j$-th node in walk sequence $S$. Given an $S$ and the probability $p_{1}$, for a target node $v_{i}=S_{j}$, we sample its context $c$ in a positive pair $\left(v_{i}, v_{c}, 1\right)$ from the set $\left\{S_{k}:|j-k|<w\right\}$ with 
a probability $p_{1}$, where $w$ is a window size parameter. With a probability ${ }_{225}\left(1-p_{1}\right)$, we uniformly sample from all nodes excluding $S_{j}$ neighborhood nodes set $\left\{V \backslash\left\{S_{k}:|j-k|<w\right\}\right\}$ to obtain a $v_{\bar{c}}$ in negative pairs $\left(v_{i}=S_{j}, v_{\bar{c}}, 0\right)$. After sampling is finished, all sampled triplets are shuffled to avoid that the order of the samples holds any significance.

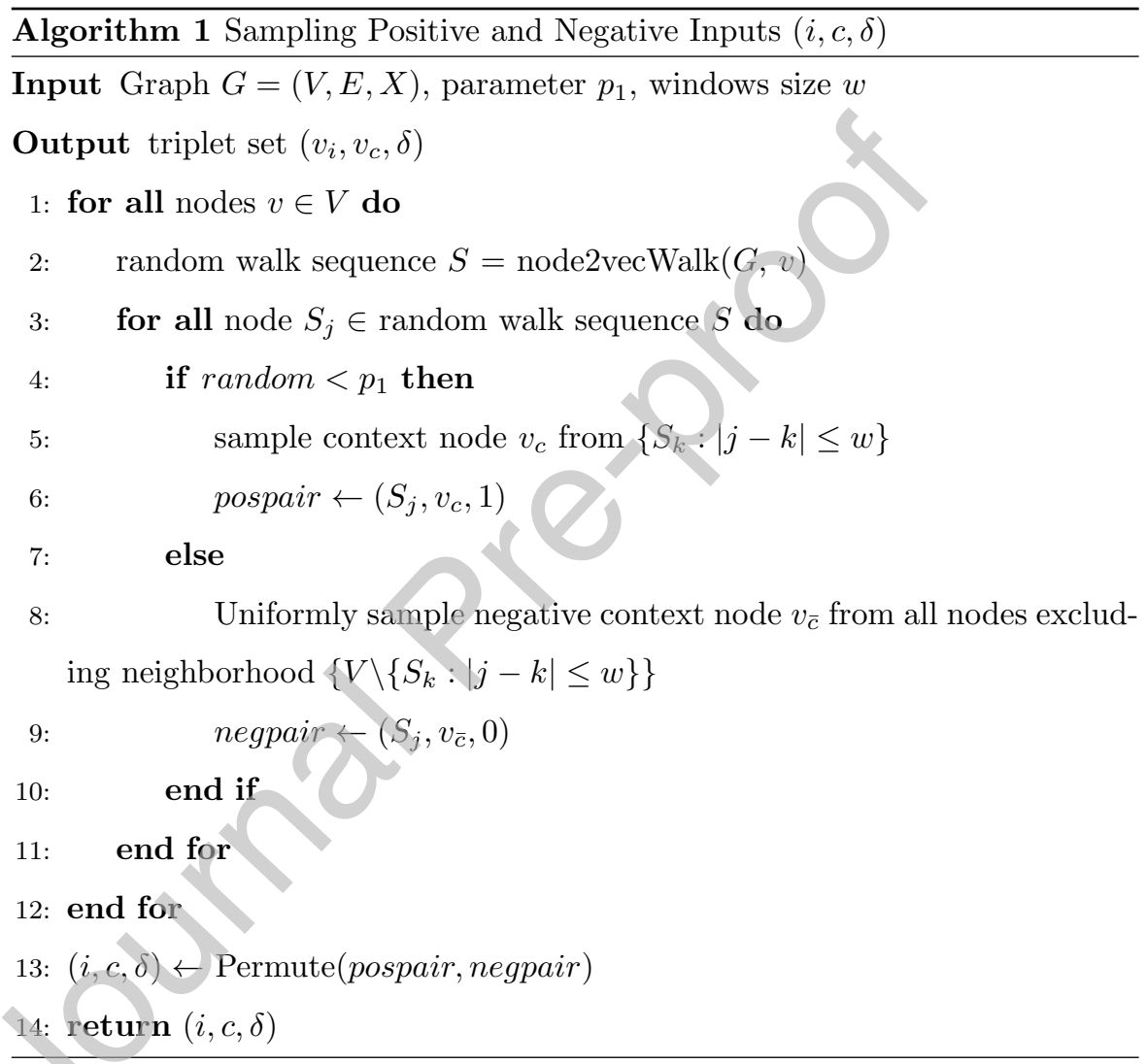

\subsection{Siamese Architecture}

A Siamese neural network consists of twin neural networks, accepting two inputs that are joined by a unified energy function at the top, as illustrated in Figure 1. Each subnetwork of the twin neural networks is a multi-layer perceptron (MLP) with non-linear activation functions. The subnetwork's output is the representation of its input (by a non-linear mapping). The cost function 
defined at the top is to compare the representations of two inputs, forcing positive input pairs to have similar representations and negative input pairs to have different representations. Since our sampled positive and negative pairs encode network topology information, by taking node contents of a positive/negative pair as inputs, Siamese architecture is a natural choice for integrating the network contents and topology,

Formally, given an input triplet $\left(v_{i}, v_{c}, \delta\right)$ (sampled in Sec. 3.3), the two nodes $v_{i}$ and $v_{c}$ go through two MLP subnetworks separately. The outputs of these subnetworks are fed to the top layer with an energy function measuring the output difference. The training target of the Siamese network is to minimize the squared Euclidean distance between the final representation of similar (positive) pairs and maximize the distance for dissimilar (negative) pairs.

We train the subnetworks of Siamese network as a deep neural network that parametrically learns the node representation based on the node content features. Given the triplet $\left(v_{i}, v_{c}, \delta\right)$, the input features for both subnetworks are the corresponding content features $X_{i}$ for target node $v_{i}$ and $X_{c}$ for context node $v_{c}$. Here we use the multi-layer perceptron (MLP) in the twin network to serve as $f_{\theta}$ where the $h$-th hidden layer is given by

$$
z_{h}\left(X_{i}\right)=g_{h}\left(W_{h} \cdot z_{h-1}\left(X_{i}\right)+b_{h}\right), \quad 1 \leq h \leq H,
$$

where $g(\cdot)$ is the element-wise activation function (e.g. Sigmoid). The output of $H$-th hidden layer is the final node representation, where we use linear mapping as $z_{H}\left(X_{i}\right)=W_{H} \cdot z_{H-1}\left(X_{i}\right)+b_{H}$. We refer the MLP as the parametric mapping, denoted by $f_{\theta}(\cdot)=z_{H}(\cdot)$, and the node representation for node $v$ will be $f_{\theta}\left(X_{v}\right)$.

We define the energy function on the top to be the Euclidean distance of the target and context node in the representation space, between $f_{\theta}\left(X_{i}\right)$ and $f_{\theta}\left(X_{c}\right)$. The aim of Siamese is to make the positive node pairs nearby and negative node pairs further apart in the representation space. According to homophily in networks [22], nodes nearby tends to have similar contents, while negative node pairs are distant and/or may have distinct contents. For a triplet 


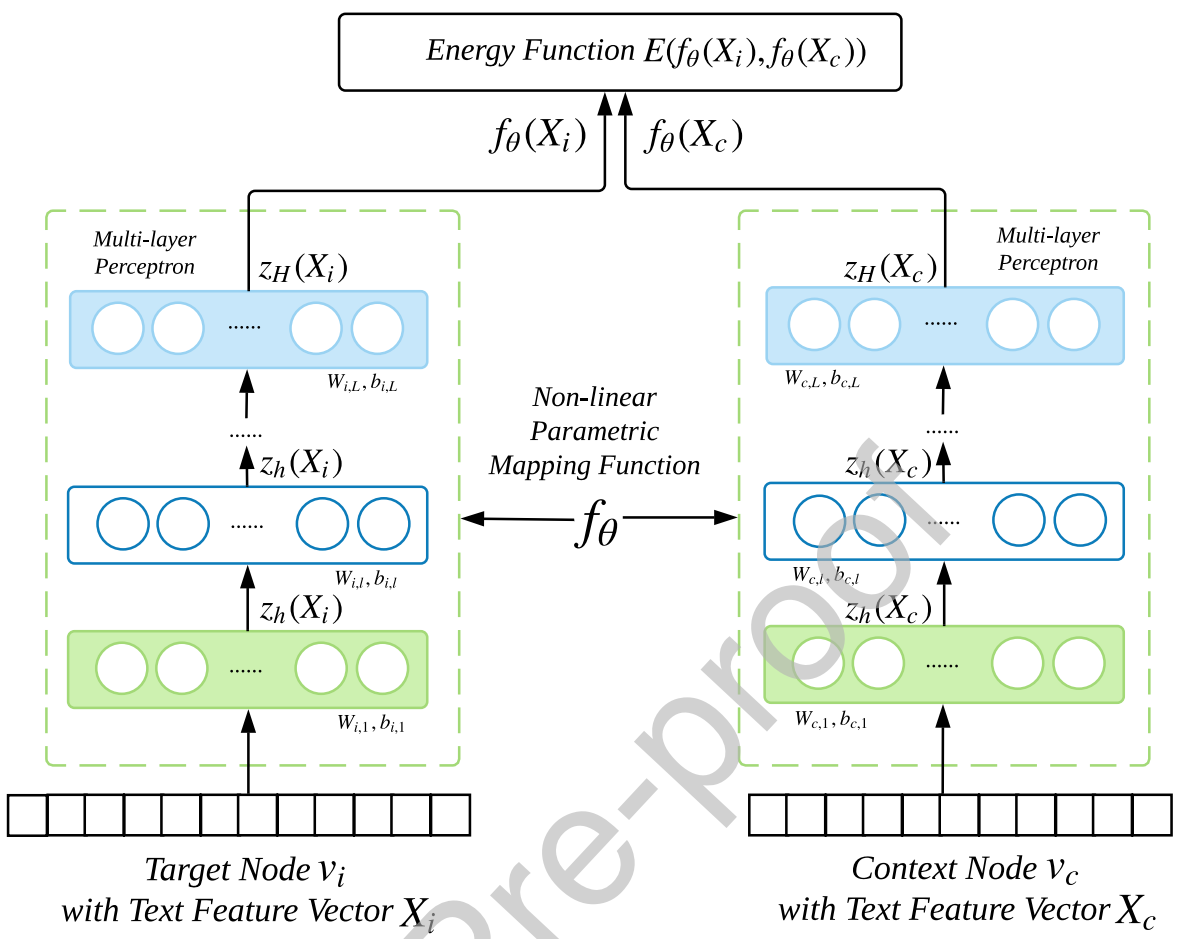

Figure 1: The Siamese architecture for parametric graph learning. Non-linear function $f_{\theta}$ read distinct node content features and joined by an energy function at the top. Energy function will use the the similarity metric.

$\left(v_{i}, v_{c}, \delta\right)$, the energy function for the positive node pair $(\delta=1)$ and negative node pair $(\delta=0)$ are defined respectively as:

$$
\begin{aligned}
& E\left(f_{\theta}\left(X_{i}\right), f_{\theta}\left(X_{c}\right), 1\right)=\left\|f_{\theta}\left(X_{i}\right)-f_{\theta}\left(X_{c}\right)\right\|_{2}, \\
& E\left(f_{\theta}\left(X_{i}\right), f_{\theta}\left(X_{c}\right), 0\right)=\max \left(0, C-\left\|f_{\theta}\left(X_{i}\right)-f_{\theta}\left(X_{c}\right)\right\|_{2}\right) .
\end{aligned}
$$

$E(\cdot, \cdot, 1)$ forces a positive node pair $\left(v_{i}, v_{c}, 1\right)$ be close, while $E(\cdot, \cdot, 0)$ makes a negative pair $\left(v_{i}, v_{c}, 0\right)$ avoid being closer than a margin $C . C$ is a hyperparameter, controlling the ideal margin for the negative pair. If a negative pair $\left(v_{i}, v_{c}\right)$ is already separated by the margin $C$, the pair will not receive any penalty and energy $E\left(f_{\theta}\left(X_{i}\right), f_{\theta}\left(X_{c}\right), 0\right)=0$. Our target is to minimize total 
loss function over the triplet set $T=\left\{\left(X_{i}^{(m)}, X_{c}^{(m)}, \delta^{(m)}\right)\right\}$ as:

$$
\mathcal{L}(T)=\sum_{m} E\left(f_{\theta}\left(X_{i}^{(m)}\right), f_{\theta}\left(X_{C}^{(m)}\right), \delta^{(m)}\right) .
$$

We introduce a hyper-parameter $\alpha \in(0,1)$ to balance the loss imposed by positive and negative samples, since the unbalanced ratio between these two populations may make the learning less effective. $\alpha$ will control the loss function to concern more about positive loss or negative loss. Finally, derived from Eq. (5), our target is to minimize the loss function with the form:

$$
\begin{aligned}
\mathcal{L}(T)= & \alpha \sum_{m: \delta^{(m)}=1} E\left(f_{\theta}\left(X_{i}^{(m)}\right), f_{\theta}\left(X_{c}^{(m)}\right), 1\right) \\
& +(1-\alpha) \sum_{m: \delta^{(m)}=0} E\left(f_{\theta}\left(X_{i}^{(m)}\right), f_{\theta}\left(X_{c}^{(m)}\right), 0\right),
\end{aligned}
$$

where the first term is the loss for all positive pairs and the second term is the loss for all negative pairs.

\subsection{Discussion}

\subsubsection{Transductive and Inductive Setting}

Our approach is flexible for generating embedding vectors for graph nodes in both transductive and inductive setting. When all nodes in the network are presented in the training phase (transductive setting), embedding vectors for all nodes are obtained by solving optimization problem defined in Eq. (6). In inductive setting, the embedding vectors of unseen nodes can be obtained by the trained MLP. To be more specific, the representation of node $v_{i}$ with content feature $X_{i}$ is the final layer's output of MLP $z_{H}\left(X_{i}\right)$ as given by Eq. (3).

While most graph representation learning methods are designed for transductive learning, there are a few inductive learning approaches like SDNE [46] and GraphSAGE [20]. These approaches take the topology information of the newly added nodes to generate the embedding vectors. For newly added nodes that only have single or no connection to existing graph, SDNE and GraphSAGE have difficulties to produce good representation for them. In contrast, GraPASA can better handle such scenarios by learning the parametric mapping 


\subsubsection{Complexity}

In the training phase, we use mini-batch gradient descent to train the variables in the Siamese architecture. We first sample a batch of triplets $\left(v_{i}, v_{c}, \delta\right)$ and then take a gradient step to optimize the loss function Eq. (6). Following the sampling method in node2vec, the time complexity is $O(|V|)$. The computation of each pair of nodes is fully independent and can easily be parallelized. Therefore, the training phase computational complexity can be considered as constant. Hence, the total complexity of GraPASA is $O(|V|)$, which is linear to the number of nodes.

\section{Experiments}

In this section, we evaluate the node representation learned by GraPASA in tasks of node classification, community detection, and representation visualization. In the node classification task, the Micro-F1 of both transductive and inductive node classification are used for quantitatively measuring the quality of learned representations. GraPASA is compared with several baseline methods on producing high-quality node representations.

\subsection{Experimental Settings}

\subsubsection{Datasets}

The proposed GraPASA is designed to learn from the network structures as well as the node content features. We select several citation networks and information networks as our experimental datasets, due to the content feature is available or not. They are Pubmed used in [49], Cora and Wiki used in [50] and Protein network used in [20].

- Cora: Cora dataset is a research paper citation network. It contains 2,708 scientific publications, which are categorized into seven classes. Each publication in the dataset is described by a 0/1-valued word vector indicating the absence/presence of the corresponding word in the dictionary. The dictionary contains 1,433 unique words. 
- Pubmed: Pubmed is very similar to Cora dataset but larger. It contains 19,717 scientific publications from three classes. Each publication is also described by a 0/1-valued word vector, while the dictionary for Pubmed dataset contains 500 unique words.

- Wiki: Wiki dataset is different from both Pubmed and Cora. It contains 2,405 web pages categorized into seventeen classes. Wiki dataset stores the web page content by using the term frequency-inverse document frequency (TF-IDF) matrix. The TF-IDF matrix contains 4,973 columns, indicating 4,973 unique words.

- PPI: PPI dataset is the protein-protein interaction dataset. The dataset contains 56,944 nodes and 818, 716 links, and is a multi-label dataset which contains 121 labels and 36.9 labels for each node. The PPI dataset uses positional gene sets, motif gene sets and immunological signatures as features vector with the dimension as 50 .

The details of dataset statistics are summarized in Table 1. The $|V|$ and $|E|$ denotes number of nodes and edges. $\operatorname{dim}(X)$ is dimension of content feature of each dataset. The feature forms the node content feature $X$. The No. of labels shows the number of labels for nodes in each data set.

We regard all three networks as undirected networks.

Table 1: Dataset statistics

\begin{tabular}{c|cccc}
\hline \hline Dataset & Cora & Pubmed & Wiki & PPI \\
\hline Content & Binary & Binary & TF-IDF & Binary \\
\hline Network Type & Citation & Citation & Hyperlink & Tissue \\
\hline$|V|$ & 2,708 & 19,717 & 2.405 & 56,944 \\
\hline$|E|$ & 5,429 & 44,338 & 17,981 & 818,716 \\
\hline $\operatorname{dim}(X)$ & 1,433 & 500 & 4,973 & 50 \\
\hline No. labels & 7 & 3 & 17 & 121 \\
\hline \hline
\end{tabular}




\subsubsection{Baseline Methods}

To validate the performance of GraPASA, we compare our model with several baseline methods, including Node2Vec, TADW, PLANETOID, GraphSAGE, Graph2Gauss, pRBM, etc. There are many other network embedding methods without consideration, because their performances are inferior to these baselines as shown in corresponding papers. The baseline methods are as follows:

- Node2Vec [19]. Node2Vec develops a second-order random walk to sample data, and uses skip-gram to learn graph embeddings.

- TADW [49]. TADW is a transductive learning approach via matrix factorization, where all nodes are observed in training phase. TADW fuses the node content and network structure together.

- Naive Combination (NaiveComb). The straightforward way to integrate graph topology and node content feature is to concatenate the Node2Vec [19] embedding vector with node content for a node.

- PLANETOID-I [50]. An inductive graph representation method with the consideration of label information. To make a fair comparison in unsupervised setting, labeling information will be removed in the training phase.

- PLANETOID-T [50]. An transductive graph representation method with consideration the labeling information. The label information is also removed here.

GraphSAGE [20]. Inductive learning structure with neighborhood aggregator. The unsupervised version is utilized for comparison.

- pRBM [48]. A parametric graph representation learning method using restricted Boltzmann machine as the generative network model.

- Graph2Gauss [4]. A parametric representation learning method to embed graph vertex as a Gaussian distribution.

- GraPASA. This is our proposed method, which fits to both transductive and inductive learning. 


\subsubsection{Parameter Settings}

For most of baseline methods, we follow the default setting in the literature. For Node2Vec, the window size $s$, walk length $l$ and number of walkers $\eta$ is set as 10, 80, 10, respectively. For Naive Combination, we concatenate the content and Node2Vec feature with 128 dimensions to form 256 dimension representation vector. For PLANETOID and GraphSAGE, both learning rates are set as 0.1. The node representation dimension $d$ is set to 256 for all experimental methods for fair comparison.

For GraPASA, we use the sigmoid activations as described in section 3.4. $\alpha$ is set to 0.3 , the random walk parameters are default parameters in Node2Vec. The layer of MLP $H$ is set to 6 for Cora, Pubmed and PPI, and 7 for Wiki dataset. For the first $H-1$ layers, Sigmoid activations and batch normalization are utilized. For the final layer, we don't use any activation or normalization techniques. We use Adagrad optimizer with learning rate as 0.001. Also earlystopping strategy is used with tolerance as 50. The sensitivity of important parameters such as dimensionality, $\alpha$ and context window size is given in section 4.4 .

\subsection{Node Classification}

We first evaluate the proposed method GraPASA in a node classification task. This is a common graph representation evaluation task, and widely used in $[50,19,20]$. For all models, we use one-vs-rest Support Vector Machine classifier implemented by scikit-learn [38].

We evaluate methods in both transductive and inductive node classification tasks. For transductive learning task, all nodes are observed during the training phase, graph embeddings are learned first, and then the classification task will range the training ratio from $10 \%$ to $90 \%$ for evaluation with embeddings. For inductive learning task, to evaluate comprehensively, the ratio of observable nodes in training will range from $10 \%$ to $90 \%$, and unobservable nodes in training are used for classification evaluation. 
We evaluate the performance of classification by both Micro-F1 and MacroF1. However, due to the limitation of space and the similar behavior of two measures, we only report Micro-F1 metric here. To ensure the reliability, we repeat the experiments for ten times, and the report average performance for each method. Also, we used the two-tailed Wilcoxon's signed rank test [15] to tell whether there is statistically significant evidence that GraPASA outperforms other methods. If no statistically significant evidence that one method outperforms the other at the $95 \%$ confidence level, the test gives p-value larger than 0.05. The experimental results are listed in Table $2-8$. Numbers in bold indicates the best performance in the corresponding row. The second best results are marked with stars. We also list the Wilcoxon's p-value from the test between GraPASA and other methods in brackets

\subsubsection{Transductive Experiments}

The transductive node classification results are shown in Table 2, 3 and 4 for Cora, Pubmed and Wiki dataset, respectively. The left column indicates the training ratio from $10 \%$ to $90 \%$.

In Cora dataset, GraPASA consistently outperforms all other methods in the Micro-F1 scores. GraPASA uses a six-layer MLP $(H=6)$ as described in Section 3.4. The output layer contains 256 units, thus produces node representation in 256-dimension.

GraPASA is built upon Node2Vec method with additional graph node content features. Comparing to transductive network learning methods with only topology feature (Node2Vec), most of methods with both network topology and content feature will obtain better performance. TADW and NaiveComb can be regarded as two linear combination methods that fuse topology and content feature. GraPASA can outperform both TADW and NaiveComb for the non-linear mapping feature. Also, comparing to two non-linear network embedding methods, PLANETOID-T and pRBM, our approach can obtain better performance because we used deeper neural network than both methods. Meanwhile, all the p-value in the table is smaller than 0.05 , which gives the statistically significant 
Table 2: Micro-F1 (\%) and statistical test results of Transductive Multi-class Classification on the Cora Network Dataset

\begin{tabular}{|c|c|c|c|c|c|c|}
\hline \% Labeled & Planetoid-T & Node2Vec & TADW & NaiveComb & $\mathrm{pRBM}$ & GraPASA \\
\hline \multirow{2}{*}{$\begin{array}{c}10 \% \\
\text { (p-value) }\end{array}$} & 74.52 & 77.44 & 82.32 & 79.38 & 82.40 & 84.22 \\
\hline & $(0.0001)$ & $(0.0001)$ & $(0.0036)$ & $(0.0001)$ & $(0.0006)$ & \\
\hline \multirow{2}{*}{$\begin{array}{c}20 \% \\
\text { (p-value) }\end{array}$} & 78.59 & 80.85 & 84.95 & 81.18 & 82.38 & 85.66 \\
\hline & $(0.0001)$ & $(0.0001)$ & $(0.0353)$ & $(0.0001)$ & $(0.0001)$ & \\
\hline \multirow{2}{*}{$\begin{array}{c}30 \% \\
\text { (p-value) }\end{array}$} & 79.35 & 83.70 & 85.99 & 83.21 & 83.24 & 86.09 \\
\hline & $(0.0001)$ & $(0.0003)$ & $(0.8227)$ & $(0.0001)$ & $(0.0001)$ & \\
\hline \multirow{2}{*}{$\begin{array}{c}40 \% \\
\text { (p-value) }\end{array}$} & 80.47 & 83.38 & 86.03 & 84.11 & 83.31 & 86.93 \\
\hline & $(0.0001)$ & $(0.0001)$ & $(0.0492)$ & $(0.0001)$ & $(0.0001)$ & \\
\hline \multirow{2}{*}{$\begin{array}{c}50 \% \\
\text { (p-value) }\end{array}$} & 82.91 & 83.97 & 86.93 & 84.06 & 83.69 & 87.66 \\
\hline & $(0.0001)$ & $(0.0001)$ & $(0.0511)$ & $(0.0001)$ & $(0.0001)$ & \\
\hline \multirow{2}{*}{$\begin{array}{c}60 \% \\
\text { (p-value) }\end{array}$} & 84.85 & 84.59 & 87.31 & 85.74 & 84.09 & 88.65 \\
\hline & $(0.0001)$ & $(0.0001)$ & $(0.0253)$ & $(0.0001)$ & $(0.0001)$ & \\
\hline \multirow{2}{*}{$\begin{array}{c}70 \% \\
\text { (p-value) }\end{array}$} & 85.12 & 84.69 & 87.74 & 86.40 & 84.23 & 88.84 \\
\hline & $(0.0001)$ & $(0.0001)$ & $(0.0235)$ & $(0.0007)$ & $(0.0001)$ & \\
\hline \multirow{2}{*}{$\begin{array}{c}80 \% \\
\text { (p-value) }\end{array}$} & 85.87 & 84.87 & 88.46 & 85.93 & 85.09 & 88.93 \\
\hline & $(0.0002)$ & $(0.0001)$ & $(0.1560)$ & $(0.0001)$ & $(0.0001)$ & \\
\hline \multirow{2}{*}{$\begin{array}{c}90 \% \\
\text { (p-value) }\end{array}$} & 86.61 & 85.21 & 87.08 & 86.25 & 85.90 & 89.80 \\
\hline & $(0.0003)$ & $(0.0001)$ & $(0.0001)$ & $(0.0001)$ & $(0.0001)$ & \\
\hline
\end{tabular}

evidence that GraPASA outperforms all other methods.

In the Pubmed dataset, GraPASA outperforms other methods as well. Since Pubmed dataset has a smaller number of node content features than Cora (500 vs 1433$)$, we used a six-layer MLP $(H=6)$ as described in Section 3.4. The number of output units is also set to 256 .

According to Table 3, using only the naive combination of content and topology feature can make a good representation. Some non-linear representation methods such as PLANETOID and pRBM do not learn a better representation. This might be due to the shallow network architecture which cannot model the complex mapping. Also, appropriate integration of two types of information is important. Otherwise, they may not complement each other but cause conflicts.

However, GraPASA takes advantages of both types of information and outper- 
Table 3: Micro-F1 (\%) and statistical test results of Transductive Multi-class Classification on the Pubmed Network Dataset

\begin{tabular}{|c|c|c|c|c|c|c|}
\hline \% Labeled & Planetoid-T & Node2Vec & TADW & NaiveComb & $\mathrm{pRBM}$ & GraPASA \\
\hline \multirow{2}{*}{$\begin{array}{c}10 \% \\
\text { (p-value) }\end{array}$} & 77.83 & 79.35 & 82.12 & 81.80 & 81.58 & 86.61 \\
\hline & $(0.0001)$ & $(0.0001)$ & $(0.0001)$ & $(0.0001)$ & $(0.0001)$ & \\
\hline \multirow{2}{*}{$\begin{array}{c}20 \% \\
\text { (p-value) }\end{array}$} & 79.59 & 80.11 & 83.21 & 83.40 & 81.70 & 87.06 \\
\hline & $(0.0001)$ & $(0.0001)$ & $(0.0001)$ & $(0.0001)$ & $(0.0001)$ & \\
\hline \multirow{2}{*}{$\begin{array}{c}30 \% \\
\text { (p-value) }\end{array}$} & 81.75 & 80.33 & 83.37 & 84.48 & 81.75 & 87.46 \\
\hline & $(0.0001)$ & $(0.0001)$ & $(0.0001)$ & $(0.0002)$ & $(0.0001)$ & \\
\hline \multirow{2}{*}{$\begin{array}{c}40 \% \\
\text { (p-value) }\end{array}$} & 82.89 & 80.45 & 84.33 & 85.48 & 81.88 & 87.71 \\
\hline & $(0.0001)$ & $(0.0001)$ & $(0.0001)$ & $(0.0009)$ & $(0.0001)$ & \\
\hline \multirow{2}{*}{$\begin{array}{c}50 \% \\
\text { (p-value) }\end{array}$} & 83.84 & 80.94 & 84.36 & 86.16 & 82.34 & 87.76 \\
\hline & $(0.0001)$ & $(0.0001)$ & $(0.0001)$ & $(0.0111)$ & $(0.0001)$ & \\
\hline \multirow{2}{*}{$\begin{array}{c}60 \% \\
\text { (p-value) }\end{array}$} & 83.95 & 81.27 & 84.64 & 86.43 & 82.71 & 87.85 \\
\hline & $(0.0001)$ & $(0.0001)$ & $(0.0005)$ & $(0.0186)$ & $(0.0001)$ & \\
\hline \multirow{2}{*}{$\begin{array}{c}70 \% \\
\text { (p-value) }\end{array}$} & 85.21 & 81.35 & 84.34 & 86.71 & 82.88 & 87.96 \\
\hline & $(0.0002)$ & $(0.0001)$ & $(0.0001)$ & $(0.0137)$ & $(0.0001)$ & \\
\hline \multirow{2}{*}{$\begin{array}{c}80 \% \\
\text { (p-value) }\end{array}$} & 85.98 & 81.74 & 85.30 & 87.52 & 83.60 & 88.75 \\
\hline & $(0.0001)$ & $(0.0001)$ & $(0.0001)$ & $(0.0206)$ & $(0.0001)$ & \\
\hline \multirow{2}{*}{$\begin{array}{c}90 \% \\
\text { (p-value) }\end{array}$} & 86.75 & 81.90 & 85.90 & 87.78 & 83.74 & 88.75 \\
\hline & $(0.0009)$ & $(0.0001)$ & $(0.0001)$ & $(0.0333)$ & $(0.0001)$ & \\
\hline
\end{tabular}

forms all other methods in all cases. Meanwhile, all the p-value is smaller than 0.05 as well, which proves that GraPASA outperforms all other methods.

In the Wiki dataset, GraPASA basically outperforms other approaches. For the dataset, as node content feature is presented in TF-IDF and more complex than that in Cora and Pubmed, we used a seven-layer MLP $(H=7)$ as described in Section 3.4. The number of units in the output layer is also set to 256 .

In this dataset, the pRBM and PLANETOID can fit to learn more sophisticated content features and provide better performance comparing to non-linear methods, such as TADW and naive combination. GraPASA can also better fit the sophisticated content feature for its multi-layer perceptron non-linear mapping, and obtain higher classification Micro-F1 against other baseline methods.

In the PPI dataset, the dimension of content vector is only 50 , so we use a 
Table 4: Micro-F1 (\%) and statistical test results of Transductive Multi-class Classification on the Wiki Network Dataset

\begin{tabular}{|c|c|c|c|c|c|c|}
\hline \% Labeled & Planetoid-T & Node2Vec & TADW & NaiveComb & $\mathrm{pRBM}$ & GraPASA \\
\hline \multirow{2}{*}{$\begin{array}{c}10 \% \\
\text { (p-value) }\end{array}$} & 68.77 & 60.09 & 72.66 & 63.18 & 72.21 & 74.49 \\
\hline & $(0.0001)$ & $(0.0001)$ & $(0.0008)$ & $(0.0001)$ & $(0.0003)$ & \\
\hline \multirow{2}{*}{$\begin{array}{c}20 \% \\
\text { (p-value) }\end{array}$} & 73.29 & 64.76 & 74.54 & 68.01 & 73.74 & 77.58 \\
\hline & $(0.0001)$ & $(0.0001)$ & $(0.0002)$ & $(0.0001)$ & $(0.0001)$ & \\
\hline \multirow{2}{*}{$\begin{array}{c}30 \% \\
\text { (p-value) }\end{array}$} & 74.85 & 66.51 & 75.83 & 72.06 & 73.87 & 78.67 \\
\hline & $(0.0001)$ & $(0.0001)$ & $(0.0001)$ & $(0.0001)$ & $(0.0001)$ & \\
\hline \multirow{2}{*}{$\begin{array}{c}40 \% \\
\text { (p-value) }\end{array}$} & 74.96 & 68.67 & 76.95 & 73.70 & 74.83 & 79.55 \\
\hline & $(0.0001)$ & $(0.0001)$ & $(0.0004)$ & $(0.0001)$ & $(0.0001)$ & \\
\hline \multirow{2}{*}{$\begin{array}{c}50 \% \\
\text { (p-value) }\end{array}$} & 75.32 & 69.19 & 77.36 & 75.10 & 75.57 & 80.13 \\
\hline & $(0.0001)$ & $(0.0001)$ & $(0.0007)$ & $(0.0001)$ & $(0.0001)$ & \\
\hline \multirow{2}{*}{$\begin{array}{c}60 \% \\
\text { (p-value) }\end{array}$} & 76.85 & 69.34 & 78.64 & 75.78 & 75.40 & 80.81 \\
\hline & $(0.0001)$ & $(0.0001)$ & $(0.0009)$ & $(0.0001)$ & $(0.0001)$ & \\
\hline \multirow{2}{*}{$\begin{array}{c}70 \% \\
\text { (p-value) }\end{array}$} & 77.21 & 70.19 & 78.79 & 76.42 & 76.95 & 81.33 \\
\hline & $(0.0001)$ & $(0.0001)$ & $(0.0007)$ & $(0.0001)$ & $(0.0001)$ & \\
\hline \multirow{2}{*}{$\begin{array}{c}80 \% \\
\text { (p-value) }\end{array}$} & 79.09 & 69.23 & 79.05 & 77.58 & 77.83 & 81.42 \\
\hline & $(0.0002)$ & $(0.0001)$ & $(0.0008)$ & $(0.0002)$ & $(0.0001)$ & \\
\hline \multirow{2}{*}{$\begin{array}{c}90 \% \\
\text { (p-value) }\end{array}$} & 79.82 & 69.29 & 79.39 & 75.93 & 77.73 & 81.85 \\
\hline & $(0.0005)$ & $(0.0001)$ & $(0.0006)$ & $(0.0001)$ & $(0.0001)$ & \\
\hline
\end{tabular}

six-layer MLP $(H=6)$ in our model. The number of units in the output layer is also set to 256. GraPASA can also obtain better performance than all other methods with almost all p-value smaller than 0.05. The PPI dataset contains lower-dimensional feature vector, which makes the mapping directly from feature vector even harder. For instance, methods like TADW and NaiveComb uses linear mapping function to obtain the final representation and performs worse than structure-based method, Node2Vec. Planetoid and pRBM obtain slightly better than Node2 Vec for they learn the non-linear representation mapping functions. However, GraPASA outperforms others methods in a significant margin, which implies that GraPASA can benefit from the deep non-linear mapping function.

Overall, GraPASA consistently outperforms other transductive methods like 
Table 5: Micro-F1 (\%) and statistical test results of Transductive Multi-class Classification on the PPI Network Dataset

\begin{tabular}{|c|c|c|c|c|c|c|}
\hline \% Labeled & Planetoid-T & Node2Vec & TADW & NaiveComb & pRBM & GraPASA \\
\hline \multirow{2}{*}{$\begin{array}{c}10 \% \\
\text { (p-value) }\end{array}$} & 44.88 & 44.66 & 39.16 & 43.67 & 42.77 & 45.46 \\
\hline & $(0.0400)$ & $(0.0187)$ & $(0.0001)$ & $(0.0046)$ & $(0.0001)$ & \\
\hline \multirow{2}{*}{$\begin{array}{c}20 \% \\
\text { (p-value) }\end{array}$} & 45.91 & 44.74 & 39.79 & 43.75 & 42.87 & 46.63 \\
\hline & $(0.0333)$ & $(0.0002)$ & $(0.0001)$ & $(0.0001)$ & $(0.0001)$ & \\
\hline \multirow{2}{*}{$\begin{array}{c}30 \% \\
\text { (p-value) }\end{array}$} & 46.05 & 44.88 & 40.43 & 43.88 & 42.82 & 46.68 \\
\hline & $(0.0420)$ & $(0.0002)$ & $(0.0001)$ & $(0.0001)$ & $(0.0001)$ & \\
\hline \multirow{2}{*}{$\begin{array}{c}40 \% \\
\text { (p-value) }\end{array}$} & 46.23 & 44.89 & 40.56 & 44.05 & 42.99 & 46.98 \\
\hline & $(0.0619)$ & $(0.0001)$ & $(0.0001)$ & $(0.0002)$ & $(0.0001)$ & \\
\hline \multirow{2}{*}{$\begin{array}{c}50 \% \\
\text { (p-value) }\end{array}$} & 46.49 & 45.23 & 41.32 & 44.19 & 43.16 & 47.33 \\
\hline & $(0.0064)$ & $(0.0002)$ & $(0.0001)$ & $(0.0001)$ & $(0.0001)$ & \\
\hline \multirow{2}{*}{$\begin{array}{c}60 \% \\
\text { (p-value) }\end{array}$} & 46.81 & 45.38 & 41.40 & 44.42 & 43.35 & 47.47 \\
\hline & $(0.0400)$ & $(0.0001)$ & $(0.0001)$ & $(0.0001)$ & $(0.0001)$ & \\
\hline \multirow{2}{*}{$\begin{array}{c}70 \% \\
\text { (p-value) }\end{array}$} & 46.96 & 45.50 & 41.81 & 44.77 & 43.59 & 48.17 \\
\hline & $(0.0008)$ & $(0.0001)$ & $(0.0001)$ & $(0.0001)$ & $(0.0001)$ & \\
\hline \multirow{2}{*}{$\begin{array}{c}80 \% \\
\text { (p-value) }\end{array}$} & 47.13 & 45.63 & 41.95 & 44.96 & 43.57 & 48.23 \\
\hline & $(0.0011)$ & $(0.0001)$ & $(0.0001)$ & $(0.0001)$ & $(0.0001)$ & \\
\hline \multirow{2}{*}{$\begin{array}{c}90 \% \\
\text { (p-value) }\end{array}$} & 47.39 & 45.66 & 42.03 & 44.94 & 43.87 & 48.42 \\
\hline & $(0.0025)$ & $(0.0001)$ & $(0.0001)$ & $(0.0001)$ & $(0.0001)$ & \\
\hline
\end{tabular}

TADW, Node2Vec and pRBM on three datasets across all training ratios with almost all p-value smaller than 0.05. It demonstrates that GraPASA can fuse topology and node content feature naturally, and improve the discrimination of the generated embeddings.

\subsubsection{Inductive Experiments}

One of the advantages of our model is the capability to inductively generate graph node embedding. Here we compare our method with inductive graph representation learning method: PLANETOID-I [50], GraphSAGE [20] and Graph2Gauss [4]. The inductive node classification results are shown in Table 6,7 and 8 . The left column indicates the observable node ratio from $10 \%$ to $90 \%$. 
Table 6: Micro-F1 (\%) and statistical test results of Inductive Multi-class Classification on the Cora Network Dataset

\begin{tabular}{|c|c|c|c|c|}
\hline$\%$ Observed & Planetoid-I & GraphSAGE & Graph2Gauss & GraPASA \\
\hline \multirow{2}{*}{$\begin{array}{c}10 \% \\
\text { (p-value) }\end{array}$} & 72.11 & 80.21 & 56.65 & 81.16 \\
\hline & $(0.0001)$ & $(0.0152)$ & $(0.0001)$ & \\
\hline \multirow{2}{*}{$\begin{array}{c}20 \% \\
\text { (p-value) }\end{array}$} & 77.61 & 81.40 & 63.87 & 82.91 \\
\hline & $(0.0002)$ & $(0.0036)$ & $(0.0001)$ & \\
\hline \multirow{2}{*}{$\begin{array}{c}30 \% \\
\text { (p-value) }\end{array}$} & 79.18 & 82.67 & 67.17 & 83.77 \\
\hline & $(0.0001)$ & $(0.0169)$ & $(0.0001)$ & \\
\hline \multirow{2}{*}{$\begin{array}{c}40 \% \\
\text { (p-value) }\end{array}$} & 80.29 & 83.36 & 68.84 & 84.50 \\
\hline & $(0.0001)$ & $(0.0304)$ & $(0.0001)$ & \\
\hline \multirow{2}{*}{$\begin{array}{c}50 \% \\
\text { (p-value) }\end{array}$} & 83.84 & 84.16 & 69.95 & 85.46 \\
\hline & $(0.0006)$ & $(0.0307)$ & $(0.0001)$ & \\
\hline \multirow{2}{*}{$\begin{array}{c}60 \% \\
\text { (p-value) }\end{array}$} & 83.91 & 84.43 & 70.75 & 86.55 \\
\hline & $(0.0001)$ & $(0.0010)$ & $(0.0001)$ & \\
\hline \multirow{2}{*}{$\begin{array}{c}70 \% \\
\text { (p-value) }\end{array}$} & 84.28 & 84.84 & 71.17 & 86.76 \\
\hline & $(0.0001)$ & $(0.0064)$ & $(0.0001)$ & \\
\hline \multirow{2}{*}{$\begin{array}{c}80 \% \\
\text { (p-value) }\end{array}$} & 84.50 & 85.24 & 72.36 & 86.89 \\
\hline & $(0.0001)$ & $(0.0051)$ & $(0.0001)$ & \\
\hline \multirow{2}{*}{$\begin{array}{c}90 \% \\
\text { (p-value) }\end{array}$} & 85.38 & 85.90 & 73.70 & 87.10 \\
\hline & $(0.0001)$ & $(0.0152)$ & $(0.0001)$ & \\
\hline
\end{tabular}

As shown in Table 6, GraPASA outperforms all other inductive graph embedding methods with the test p-value smaller than 0.05. In the Cora dataset, Graph2Gauss performs over $10 \%$ lower in Micro-F1. This is caused by the shallow network structure of Graph2Gauss (only one layer) that under-fits to the best mapping function. PLANETOID-I is competitive in the inductive learning task. GraPASA outperforms PLANETOID-I due to GraPASA the usage of more hidden layers and can thus fit to more complex non-linear mapping. Also, GraPASA outperforms GraphSAGE because GraPASA can avoid the impact from selecting neighborhood nodes.

In the Pubmed dataset, the dimension of content feature is fewer than other methods and there are only three labels for classification. According to Table 7, Graph2Gauss can provide much better representation in Pubmed than in Cora, 
Table 7: Micro-F1 (\%) and statistical test results of Inductive Multi-class Classification on the Pubmed Network Dataset

\begin{tabular}{|c|c|c|c|c|}
\hline$\%$ Observed & Planetoid-I & GraphSAGE & Graph2Gauss & GraPASA \\
\hline \multirow{2}{*}{$\begin{array}{c}10 \% \\
\text { (p-value) }\end{array}$} & 80.81 & 83.22 & 83.58 & 84.51 \\
\hline & $(0.0001)$ & $(0.0438)$ & $(0.0333)$ & \\
\hline \multirow{2}{*}{$\begin{array}{c}20 \% \\
\text { (p-value) }\end{array}$} & 80.92 & 83.33 & 83.87 & 85.32 \\
\hline & $(0.0001)$ & $(0.0025)$ & $(0.0035)$ & \\
\hline \multirow{2}{*}{$\begin{array}{c}30 \% \\
\text { (p-value) }\end{array}$} & 81.18 & 83.49 & 84.06 & 85.59 \\
\hline & $(0.0001)$ & $(0.0010)$ & $(0.0012)$ & \\
\hline \multirow{2}{*}{$\begin{array}{c}40 \% \\
\text { (p-value) }\end{array}$} & 81.86 & 83.40 & 84.18 & 86.31 \\
\hline & $(0.0001)$ & $(0.0002)$ & $(0.0015)$ & \\
\hline \multirow{2}{*}{$\begin{array}{c}50 \% \\
\text { (p-value) }\end{array}$} & 81.87 & 83.55 & 84.50 & 86.56 \\
\hline & $(0.0001)$ & $(0.0001)$ & $(0.0005)$ & \\
\hline \multirow{2}{*}{$\begin{array}{c}60 \% \\
\text { (p-value) }\end{array}$} & 82.65 & 83.58 & 84.60 & 86.63 \\
\hline & $(0.0001)$ & $(0.0003)$ & $(0.0009)$ & \\
\hline \multirow{2}{*}{$\begin{array}{c}70 \% \\
\text { (p-value) }\end{array}$} & 83.10 & 84.85 & 84.72 & 86.94 \\
\hline & $(0.0001)$ & $(0.0002)$ & $(0.0025)$ & \\
\hline \multirow{2}{*}{$\begin{array}{c}80 \% \\
\text { (p-value) }\end{array}$} & 83.12 & 88.24 & 84.98 & 87.05 \\
\hline & $(0.0001)$ & $(0.071)$ & $(0.0004)$ & \\
\hline \multirow{2}{*}{$\begin{array}{c}90 \% \\
(\mathrm{p} \text {-value) }\end{array}$} & 85.46 & 85.39 & 85.61 & 87.29 \\
\hline & $(0.0004)$ & $(0.0011)$ & $(0.0006)$ & \\
\hline
\end{tabular}

because Pubmed dataset has shorter content feature and the mapping could be easier to fit. The Graph2Gauss method can fit the mapping function with a single layer network. Meanwhile, PLANETOID and GraphSAGE also obtain good representation results. But Graph2Gauss outperforms other methods in general. This is because Graph2Gauss can avoid the affect by the neighborhood nodes, and the node within same community can be more separable. GraPASA follows similar metric learning paradigm and benefits from the metric learning advantage as Graph2Gauss. Also, GraPASA can outperform all other methods in the Pubmed dataset with p-value smaller than 0.05 in general.

The Wiki dataset is different because it uses the tf-idf feature vector. The result of Wiki dataset is shown in Table 8. GraPASA performs much better when less nodes observed in training. In fact, even with only $20 \%$ nodes observed, 
Table 8: Micro-F1 (\%) and statistical test results of Inductive Multi-class Classification on the Wiki Network Dataset

\begin{tabular}{|c|c|c|c|c|}
\hline$\%$ Observed & Planetoid-I & GraphSAGE & Graph2Gauss & GraPASA \\
\hline \multirow{2}{*}{$\begin{array}{c}10 \% \\
\text { (p-value) }\end{array}$} & 68.91 & 61.05 & 56.65 & 74.39 \\
\hline & $(0.0001)$ & $(0.0001)$ & $(0.0001)$ & \\
\hline \multirow{2}{*}{$\begin{array}{c}20 \% \\
\text { (p-value) }\end{array}$} & 73.54 & 64.08 & 57.41 & 77.88 \\
\hline & $(0.0001)$ & $(0.0001)$ & $(0.0001)$ & \\
\hline \multirow{2}{*}{$\begin{array}{c}30 \% \\
\text { (p-value) }\end{array}$} & 75.41 & 66.50 & 61.57 & 78.00 \\
\hline & $(0.0001)$ & $(0.0001)$ & $(0.0001)$ & \\
\hline \multirow{2}{*}{$\begin{array}{c}40 \% \\
\text { (p-value) }\end{array}$} & 75.20 & 66.94 & 64.32 & 78.25 \\
\hline & $(0.0001)$ & $(0.0001)$ & $(0.0001)$ & \\
\hline \multirow{2}{*}{$\begin{array}{c}50 \% \\
\text { (p-value) }\end{array}$} & 73.98 & 68.35 & 68.83 & 78.22 \\
\hline & $(0.0001)$ & $(0.0001)$ & $(0.0001)$ & \\
\hline \multirow{2}{*}{$\begin{array}{c}60 \% \\
\text { (p-value) }\end{array}$} & 75.75 & 70.48 & 70.69 & 78.75 \\
\hline & $(0.0001)$ & $(0.0001)$ & $(0.0001)$ & \\
\hline \multirow{2}{*}{$\begin{array}{c}70 \% \\
\text { (p-value) }\end{array}$} & 76.78 & 71.32 & 72.16 & 78.83 \\
\hline & $(0.0001)$ & $(0.0001)$ & $(0.0001)$ & \\
\hline \multirow{2}{*}{$\begin{array}{c}80 \% \\
\text { (p-value) }\end{array}$} & 78.65 & 72.72 & 73.56 & 79.94 \\
\hline & $(0.0487)$ & $(0.0001)$ & $(0.0001)$ & \\
\hline \multirow{2}{*}{$\begin{array}{c}90 \% \\
\text { (p-value) }\end{array}$} & 80.34 & 74.70 & 73.88 & 80.08 \\
\hline & $(0.4552)$ & $(0.0001)$ & $(0.0001)$ & \\
\hline
\end{tabular}

GraPASA performs better than other methods with $80 \%$ data observed. The statistical test also proved that GraPASA outperforms all other methods. This performance presents the effectiveness of GraPASA in sparsely labeled networks.

We also use PPI dataset for inductive learning. Different from the above three datasets, PPI dataset contains multiple un-connected subgraphs, which is a natural source for inductive learning. We followed the experiment settings in [20] and chose the top $k$ biggest isolated subgraphs for inductive learning.

As presented in Table 9, GraPASAcan achieve about two percent improvement against Planetoid-I, which outperforms all other inductive network representation methods, such as GraphSAGE and Graph2Gauss. The statistical test proves that GraPASA achieves better performance than other methods.

Overall, the Siamese architecture in GraPASA decently integrates the graph 
Table 9: Micro-F1 (\%) and statistical test results of Inductive Multi-class Classification on the PPI Network Dataset

\begin{tabular}{|c|c|c|c|c|}
\hline \% Unobserved graphs & Planetoid-I & GraphSAGE & Graph2Gauss & GraPASA \\
\hline \multirow{2}{*}{$\begin{array}{c}1 \\
\text { (p-value) }\end{array}$} & 46.85 & 45.39 & 43.95 & 48.34 \\
\hline & $(0.0072)$ & $(0.0001)$ & $(0.0001)$ & \\
\hline \multirow{2}{*}{$\begin{array}{c}2 \\
\text { (p-value) }\end{array}$} & 46.57 & 45.11 & 43.19 & 48.32 \\
\hline & $(0.0022)$ & $(0.0001)$ & $(0.0001)$ & \\
\hline \multirow{2}{*}{$\begin{array}{c}3 \\
\text { (p-value) }\end{array}$} & 46.33 & 45.03 & 42.94 & 48.31 \\
\hline & $(0.0015)$ & $(0.0001)$ & $(0.0001)$ & \\
\hline \multirow{2}{*}{$\begin{array}{c}4 \\
\text { (p-value) }\end{array}$} & 46.15 & 44.93 & 42.55 & 47.85 \\
\hline & $(0.0011)$ & $(0.0001)$ & $(0.0001)$ & \\
\hline \multirow{2}{*}{$\begin{array}{c}5 \\
\text { (p-value) }\end{array}$} & 46.01 & 44.75 & 42.18 & 47.78 \\
\hline & $(0.0028)$ & $(0.0003)$ & $(0.0001)$ & \\
\hline \multirow{2}{*}{$\begin{array}{c}6 \\
\text { (p-value) }\end{array}$} & 45.89 & 44.56 & 41.89 & 47.75 \\
\hline & $(0.0022)$ & $(0.0001)$ & $(0.0001)$ & \\
\hline \multirow{2}{*}{$\begin{array}{c}7 \\
\text { (p-value) }\end{array}$} & 45.93 & 44.45 & 41.62 & 47.64 \\
\hline & $(0.0028)$ & $(0.0001)$ & $(0.0001)$ & \\
\hline \multirow{2}{*}{$\begin{array}{c}8 \\
\text { (p-value) }\end{array}$} & 45.68 & 44.17 & 41.24 & 47.58 \\
\hline & $(0.0008)$ & $(0.0001)$ & $(0.0001)$ & \\
\hline \multirow{2}{*}{$\begin{array}{c}9 \\
\text { (p-value) }\end{array}$} & 45.17 & 43.89 & 41.08 & 47.48 \\
\hline & $(0.0009)$ & $(0.0001)$ & $(0.0001)$ & \\
\hline
\end{tabular}

topology and node content feature and produces effective node representation from parametric mapping. The results in both transductive and inductive node classification task show that GraPASA gain improvements over other methods.

\subsection{Community Detection}

Community detection is a common task that detects groups of nodes with similar characteristics $[35,44]$. In community detection task, the goal is to predict the most likely community assigned to each node. Since multi-class datasets had ground truth label, We can quantitatively evaluate the performance of community detection with the labels. For evaluation, we use metrics that can present the correspondence between the detected and ground-truth communities. 
Table 10: Result table for community detection using Normalized Mutual Information

\begin{tabular}{l|l|l|l}
\hline Method & Cora & Pubmed & Wiki \\
\hline \hline Node2Vec & 0.4677 & 0.2561 & 0.3842 \\
\hline TADW & 0.5657 & 0.2381 & 0.4024 \\
\hline NaiveComb & 0.4972 & 0.2565 & 0.2715 \\
\hline GraphSAGE & $0.5459^{*}$ & $0.3464^{*}$ & $0.4667^{*}$ \\
\hline Graph2Gauss & 0.3011 & 0.2803 & 0.2599 \\
\hline PLANETOID-T & 0.4637 & 0.3021 & 0.4517 \\
\hline pRBM & 0.2915 & 0.2451 & 0.1656 \\
\hline Grapasa & $\mathbf{0 . 5 8 0 4}$ & $\mathbf{0 . 3 6 0 2}$ & $\mathbf{0 . 4 8 2 4}$ \\
\hline
\end{tabular}

Formally, given the representations $f_{\theta}(X)$, we use $k$-means algorithm [26] to cluster representations for community detection. The number of clusters $K$ in $k$-means algorithm is set the same as the number of distinct labels in the dataset. By using $k$-means, each node will be labeled a detected community label $C_{i}$. The target of this task is quantifying the level of correspondence of ground truth communities $C^{*}$ and detected communities $C$.

Here we use Normalized Mutual Information (NMI) and Adjusted Rand Index as our metric, both are widely used in community detection evaluation [52]:

- $N M I$ Normalized Mutual Information follows the criterion in information theory to evaluate the difference between detected clusters and ground truth clusters. The range of $N M I$ is between 0 and 1 , higher value indicates detected cluster is more similar to ground truth.

- ARI: Adjusted Rand Index is a measure of the similarity between ground truth and detected community results. It is the corrected-for-chance version of Rand Index. This metric is also independent of labels and has value range between 0 and 1 . bers in bold indicates the best performance in the corresponding column. The 
Table 11: Result table for community detection using Adjusted Rand Index

\begin{tabular}{l|l|l|l}
\hline Method & Cora & Pubmed & Wiki \\
\hline \hline Node2Vec & 0.3892 & 0.2823 & 0.2177 \\
\hline TADW & 0.5227 & 0.2227 & 0.0787 \\
\hline Naive Combination & 0.4168 & 0.2825 & 0.0482 \\
\hline GraphSAGE & $0.4617^{*}$ & $0.3411^{*}$ & $0.2499^{*}$ \\
\hline Graph2Gauss & 0.2697 & 0.2931 & 0.1645 \\
\hline PLANETOID-T & 0.4213 & 0.2455 & 0.2335 \\
\hline pRBM & 0.2819 & 0.2329 & 0.1592 \\
\hline Grapasa & $\mathbf{0 . 5 5 0 7}$ & $\mathbf{0 . 3 5 7 8}$ & $\mathbf{0 . 2 5 5 7}$ \\
\hline
\end{tabular}

second best results are marked with stars.

In the Cora dataset, GraPASA outperforms other methods in both NMI and ARI metrics. GraphSAGE and TADW is very competitive in this dataset, both methods have similar performance as GraPASA . GraphSAGE can infer network embeddings using neighborhood nodes, which will make nodes with same label more converged comparing to others. GraPASA also will force the nodes in the neighbor closer in the representation space, but we can directly infer the embedding to avoid the variance by neighbors. So GraPASA can slightly better than GraphSAGE.

In the Pubmed dataset, only three communities in total. GraphSAGE and Graph2Gauss are very competitive. GraphSAGE takes more advantage because the aggregation of neighborhood nodes. Also, the distributional embedding of Graph2Gauss makes some hub nodes hard to be clustered to certain communities. GraPASA takes the advantage of GraphSAGE by bring topology information into the non-linear mapping function, and avoids the disadvantage of distributional representation to make embeddings easier to cluster.

In the Wiki dataset, many content feature based representation methods have very low score in NMI. This is because the Wiki dataset contains 17 labels, which is 17 communities in total. Linear embedding methods will easily 
misclassified nodes with similar content feature. Deep embedding methods, such as Node2Vec, Planetoid, and GraphSAGE, performs well in the Wiki dataset. It indicates the effectiveness of deep network models. Meanwhile, GraPASA can outperform other methods for better capturing the non-linear mapping with MLP.

The community detection results show many recent graph representation methods can deal with the graph with minor community labels, but fail to generate effective representation to deal the dataset with many distinct labels. Our approach generates more meaningful representation for community detection, especially when facing dataset with the larger number of community labels.

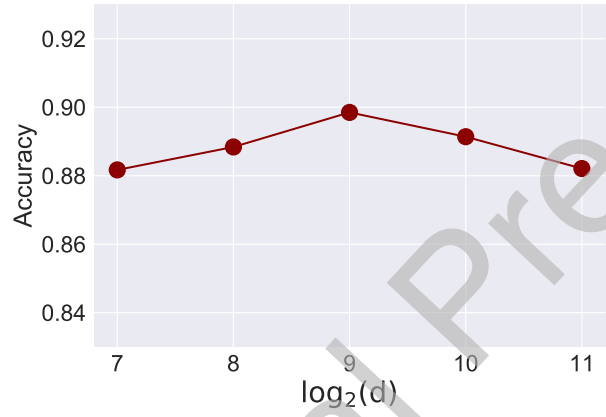

(a) Dimensions

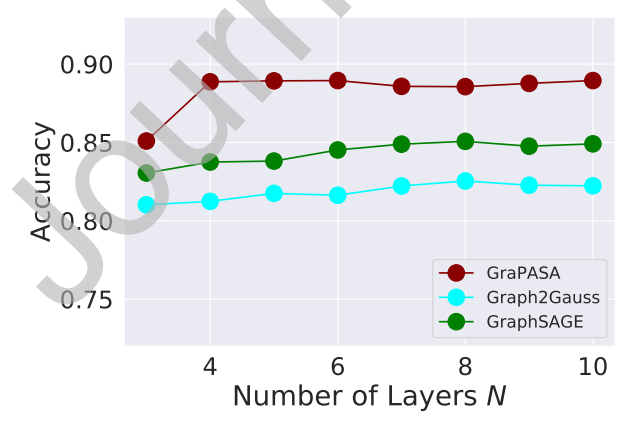

(c) Layers $N$

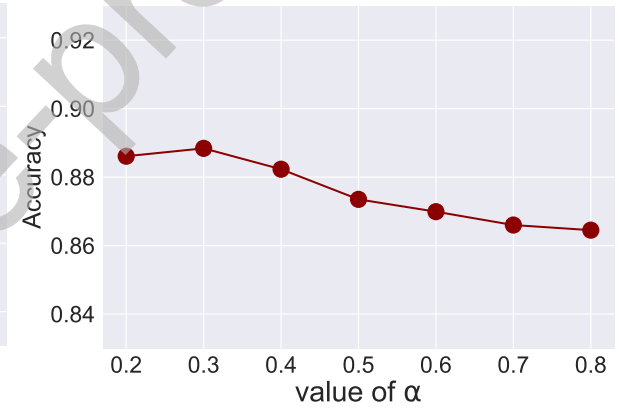

(b) Parameter $\alpha$

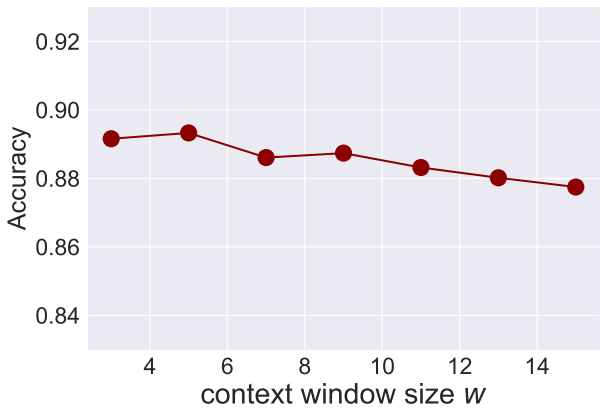

(d) Context-size

Figure 2: Parameter sensitivity analysis for GraPASA in transductive node classification task on Cora with $70 \%$ training data. 


\subsection{Parameter Sensitivity}

In this section, we investigate the performance of GraPASA w.r.t three important parameters on Cora dataset. Specifically, we examine how the representation dimension $d$, the context-window $w$ and positive-negative loss adjusted with training ratio as $70 \%$. Except for parameter being tested, all other parameters are set to default values.

First, we examine the sensitivity of representation dimension $d$ and context window size $w$. Figure 2 (a) illustrates the performance of GraPASA actually is not sensitive to dimension changes. Even though GraPASA draws the peak at dimension 512, the Micro-F1 changed only a little for higher dimensions. Figure 2 (c) indicates that number of layers for multi-layered network is not quite sensitive to number of layers. GraPASAobtains the best performance when using 6 layers, and the performance will remain steady when the number of layers increases. Meanwhile, we tested GraphSAGE and Graph2Gauss with deeper networks. These two methods can obtain higher accuracy when the number of layers increases. But GraPASA still outperform all these methods. We believe this is because Graph2Gauss and GraphSAGE uses KL-divergence and logistic loss, which are more sensitive for shallower architecture, while GraPASA uses the contrastive loss which can capture more difference in deeper networks. Figure $2(\mathrm{~d})$ presents that GraPASA is also not sensitive to context window $w$. The performance will drop when the context window increases, but only 2 percent decline emerged between $w=3$ and $w=15$

Figure 2 (b) shows that the performance of GraPASA decreases when the parameter $\alpha$ gets larger. When $\alpha$ is set to 0.3 , it produces the best trade-off between loss of positive and negative samples. In other datasets, $\alpha$ can be set by cross-validation. Usually, a small $\alpha$ (less than 0.5 ) is preferred, as negative samples are more massive than positive samples. 


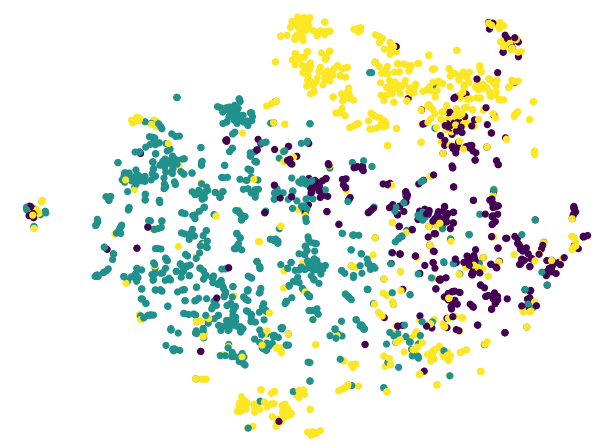

(a) Node2 Vec

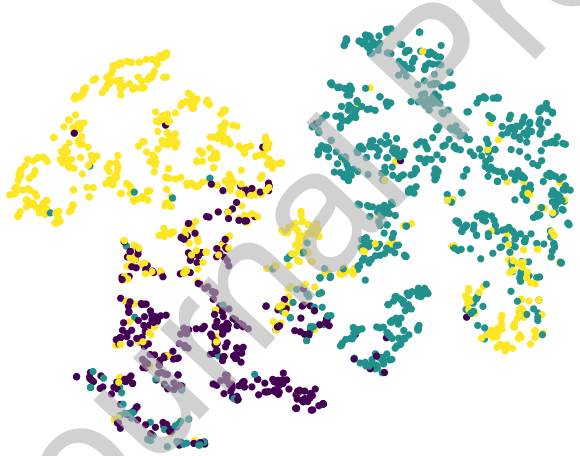

(c) GraphSAGE

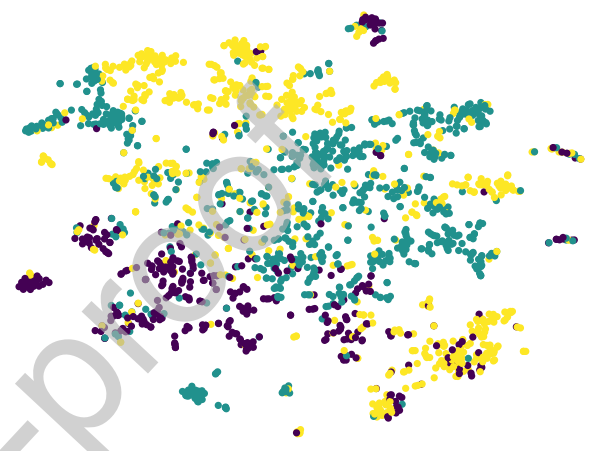

(b) TADW

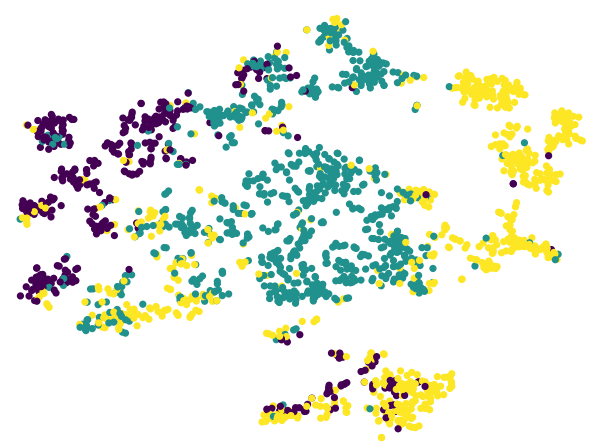

(d) GraPASA

Figure 3: Visualization of 8000 randomly selected papers in Pubmed Dataset. Nodes are colored by their ground truth labels. 


\section{5. t-SNE Visualization} ingful visualization that can map a network in a low dimensional space (usually 2d). We use t-SNE [30] to visualize representation learned by several different models. To show a clear visualization result, 8000 nodes are randomly selected from the dataset. There are only three labels in the visualization dataset.

Figure 3 illustrates the visualization of graph representations obtained from different model using t-SNE, where nodes are colored by their ground truth labels. For both Node2 Vec and TADW, they cannot show a clear boundary between each clusters. Nodes in the center are mixed with other two labels.

For the state-of-the-art GraphSAGE method, the t-SNE result is more plausible. All three categories of nodes can have a clear boundary. However, there is a narrow and long area that nodes with label yellow are intertwined with nodes with label purple. The results of the GraPASA are more separable in visualization. Since the proposed method GraPASA share the same heuristic from Skip-gram as Node2Vec, it tends to share the similar problem as node2vec: some of the high-degree nodes (associated with many edges) are mapped closely in the center area due to their hub role played in the network community. However, the results of GraPASA are more converged and separable because of the contrastive information learned in the siamese network. As a result, GraPASA performs better and generates a more meaningful layout of the network than other methods.

\section{Conclusion}

We propose a novel parametric representation learning method, GraPASA, which maps graph nodes into a low-dimensional space by integrating the network topology and node content information. We show representations learned methods, such as Node2vec, PLANETOID, GraphSAGE, Graph2Gauss, etc, in node classification, community detection and network visualization tasks. 
Parametric representation learning is a promising solution for integrating multiple types of information. In our future work, advanced models will be designed for learning more comprehensive representations from multi-source enriched networks, considering not only network topology, node content feature, but also edge content. Also, using parametric learning for the graph with special structures like the heterogeneous network will be of our interests.

\section{Declaration of Competing Interest}

The authors declare that they have no known competing financial interests or personal relationships that could have appeared to influence the work reported in this paper.

\section{Acknowledgements}

This work was partially supported and funded by King Abdullah University of Science and Technology (KAUST) through the KAUST Office of Sponsored Research (OSR) under Award No. 2639, National Key R\&D Program of China (2017YFC0803700), National Natural Science Foundation of China (61502320), Science Foundation of Shenzhen City in China (JCYJ20160419152942010), the State Key Laboratory of Software Development Environment, and Aeronautical

Science Foundation of China.

\section{References}

[1] Akujuobi, U., Yufei, H., Zhang, Q., Zhang, X., 2019. Recurrent attention walk for semi-supervised classification, in: ICDM.

[2] Belkin, M., Niyogi, P., 2001. Laplacian eigenmaps and spectral techniques for embedding and clustering, in: Advances in Neural Information Processing Systems 14, pp. 585-591.

[3] Bengio, Y., Ducharme, R., Vincent, P., Jauvin, C., 2003. A neural probabilistic language model. Journal of machine learning research 3, 1137-1155. 
[4] Bojchevski, A., Gnnemann, S., 2018. Deep gaussian embedding of graphs: ${ }_{635}$ Unsupervised inductive learning via ranking, in: International Conference on Learning Representations. URL: https://openreview.net/forum?id= r1ZdKJ-OW.

[5] Bouchard, G., Singh, S., Trouillon, T., 2015. On approximate reasoning capabilities of low-rank vector spaces. AAAI KRR .

[6] Bromley, J., Guyon, I., LeCun, Y., Säckinger, E., Shah, R., 1993. Signature verification using a siamese time delay neural network, in: Proceedings of the 6th International Conference on Neural Information Processing Systems, pp. $737-744$.

[7] Cao, S., Lu, W., Xu, Q., 2016. Deep neural networks for learning graph representations, in: Proceedings of the Thirtieth AAAI Conference on Artificial Intelligence, pp. 1145-1152.

[8] Carreira-Perpinán, M.A., Vladymyrov, M., 2015. A fast, universal algorithm to learn parametric nonlinear embeddings, in: Advances in Neural Information Processing Systems, pp. 253-261.

[9] Chang, S., Han, W., Tang, J., Qi, G.J., Aggarwal, C.C., Huang, T.S., 2015. Heterogeneous network embedding via deep architectures, in: Proceedings of the 21th ACM SIGKDD international conference on Knowledge discovery and data mining, pp. 119-128.

[10] Chen, W., Zhang, X., Wang, J., Zhang, Y., Yan, H., Li, X., 2016. Nonlinear smoothed transductive network embedding with text information, in: Asian Conference on Machine Learning, pp. 1-16.

[11] Chen, X., Yu, G., Wang, J., Domeniconi, C., Li, Z., Zhang, X., 2019a. Activehne: Active heterogeneous network embedding, in: IJCAI, pp. 21232129 .

[12] Chen, Y., Pu, J., Liu, X., Zhang, X., 2019b. Gaussian mixture embedding of multiple node roles in networks. WWW Journal . 
[13] Chopra, S., Hadsell, R., LeCun, Y., 2005. Learning a similarity metric discriminatively, with application to face verification, in: Computer Vision and Pattern Recognition, 2005. CVPR 2005. IEEE Computer Society Conference on, pp. 539-546.

[14] Dai, Q., Li, Q., Tang, J., Wang, D., 2017. Adversarial network embedding. arXiv preprint arXiv:1711.07838 .

[15] Demšar, J., 2006. Statistical comparisons of classifiers over multiple data sets. Journal of Machine learning research 7, 1-30.

[16] Feng, R., Yang, Y., Hu, W., Wu, F., Zhuang, Y., 2017. Representation learning for scale-free networks. arXiv preprint arXiv:1711.10755 .

[17] Goodfellow, I., Bengio, Y., Courville, A., 2016. Deep Learning. http: //www.deeplearningbook.org.

[18] Gori, M., Monfardini, G., Scarselli, F., 2005. A new model for learning in graph domains, in: Neural Networks, 2005. IJCNN'05. Proceedings. 2005 IEEE International Joint Conference on, IEEE. pp. 729-734.

[19] Grover, A., Leskovec, J., 2016. node2vec: Scalable feature learning for networks. Proceedings of the 22th ACM SIGKDD international conference on Knowledge discovery and data mining 2016, 855-864.

[20] Hamilton, W.L., Ying, R., Leskovec, J., 2017. Inductive representation learning on large graphs, in: Advances in Neural Information Processing Systems.

[21] Hinton, G.E., Roweis, S.T., 2003. Stochastic neighbor embedding, in: Advances in Neural Information Processing Systems, pp. 857-864.

685 [22] Kim, K., Altmann, J., 2017. Effect of homophily on network formation. Communications in Nonlinear Science and Numerical Simulation 44, 482494. 
[23] Kipf, T.N., Welling, M., 2016. Semi-supervised classification with graph convolutional networks. arXiv preprint arXiv:1609.02907 .

${ }_{690}^{6}$ [24] Levy, O., Goldberg, Y., 2014. Neural word embedding as implicit matrix factorization, in: Advances in Neural Information Processing Systems, pp. $2177-2185$.

[25] Li, J., Zhu, J., Zhang, B., 2016. Discriminative deep random walk for network classification, in: Proceedings of the 54th Annual Meeting of the Association for Computational Linguistics, ACL.

[26] Li, Z., Zhang, S., Wang, R.S., Zhang, X.S., Chen, L., 2008. Quantitative function for community detection. Physical review. E, Statistical, nonlinear, and soft matter physics 773 Pt 2, 036109.

[27] Liu, Y., Liu, Z., Chua, T.S., Sun, M., 2015. Topical word embeddings., in: AAAI Conference on Artificial Intelligence, pp. 2418-2424.

[28] van der Maaten, L., 2009. Learning a parametric embedding by preserving local structure. RBM 500, 26.

[29] van der Maaten, L., 2014. Accelerating t-sne using tree-based algorithms. JMLR 15, 3221-3245.

705 [30] Maaten, L.v.d, Hinton, G., 2008. Visualizing data using t-sne. Journal of Machine Learning Research 9, 2579-2605.

[31] Meng, Z., Liang, S., Bao, H., Zhang, X., 2019. Co-embedding attributed networks, in: WSDM, pp. 393-401.

[32] Mikolov, T., Sutskever, I., Chen, K., Corrado, G.S., Dean, J., 2013. Distributed representations of words and phrases and their compositionality, in: Advances in neural information processing systems, pp. 3111-3119.

[33] Mueller, J., Thyagarajan, A., 2016. Siamese recurrent architectures for learning sentence similarity, in: Thirtieth AAAI Conference on Artificial Intelligence. 
[34] Neculoiu, P., Versteegh, M., Rotaru, M., Amsterdam, T.B., 2016. Learning text similarity with siamese recurrent networks. ACL 2016, 148.

[35] Newman, M.E., 2006. Modularity and community structure in networks. Proceedings of the national academy of sciences 103, 8577-8582.

[36] Nickel, M., Jiang, X., Tresp, V., 2014. Reducing the rank in relational factorization models by including observable patterns, in: Advances in Neural Information Processing Systems, pp. 1179-1187.

[37] Pan, S., Wu, J., Zhu, X., Zhang, C., Wang, Y., 2016. Tri-party deep network representation, in: Proceedings of the Twenty-Fifth International Joint Conference on Artificial Intelligence, pp. 1895-1901.

[38] Pedregosa, F., Varoquaux, G., Gramfort, A., Michel, V., Thirion, B., Grisel, O., Blondel, M., Prettenhofer, P., Weiss, R., Dubourg, V., Vanderplas, J., Passos, A., Cournapeau, D., Brucher, M., Perrot, M., Duchesnay, E., 2011. Scikit-learn: Machine learning in Python. Journal of Machine Learning Research 12, 2825-2830.

[39] Perozzi, B., Al-Rfou', R., Skiena, S., 2014. Deepwalk: online learning of social representations, in: Proceedings of the 20th ACM SIGKDD international conference on Knowledge discovery and data mining, pp. 701-710.

[40] Roweis, S.T., Saul, L.K., 2000. Nonlinear dimensionality reduction by locally linear embedding. science 290, 2323-2326.

[41] Sun, G., Zhang, X., 2019. A novel framework for node/edge attributed graph embedding, in: PAKDD 2019, pp. 169-182.

[42] Tang, J., Qu, M., Wang, M., Zhang, M., Yan, J., Mei, Q., 2015. Line: Large-scale information network embedding, in: Proceedings of the 24th International Conference on World Wide Web, pp. 1067-1077.

740 [43] Tang, L., Liu, H., 2009. Relational learning via latent social dimensions, in: Proceedings of the 15th ACM SIGKDD international conference on Knowledge discovery and data mining, pp. 817-826. 
[44] Tsitsulin, A., Mottin, D., Karras, P., Müller, E., 2018. Verse: Versatile graph embeddings from similarity measures, in: Proceedings of the 2018 World Wide Web Conference on World Wide Web, International World Wide Web Conferences Steering Committee. pp. 539-548.

[45] Tu, C., Zhang, W., Liu, Z., Sun, M., 2016. Max-margin deepwalk: discriminative learning of network representation, in: Proceedings of the TwentyFifth International Joint Conference on Artificial Intelligence (IJCAI 2016), pp. 3889-3895.

[46] Wang, D., Cui, P., Zhu, W., 2016a. Structural deep network embedding, in: Proceedings of the 22nd ACM SIGKDD International Conference on Knowledge Discovery and Data Mining, pp. 1225-1234.

[47] Wang, D., Cui, P., Zhu, W., 2016b. Structural deep network embedding, in: Proceedings of the 22th ACM SIGKDD international conference on Knowledge discovery and data mining, pp. 1225-1234.

[48] Wang, S., Tang, J., Morstatter, F., Liu, H., 2016c. Paired restricted boltzmann machine for linked data, in: Proceedings of the 25th ACM conference on Information and knowledge management, ACM. pp. 1753-1762.

[49] Yang, C., Liu, Z., Zhao, D., Sun, M., Chang, E.Y., 2015. Network representation learning with rich text information, in: Twenty-Fourth International Joint Conference on Artificial Intelligence.

[50] Yang, Z., Cohen, W.W., Salakhutdinov, R., 2016. Revisiting semisupervised learning with graph embeddings, in: Proceedings of The 33rd International Conference on Machine Learning, pp. 40-48.

[51] Zhang, D., Yin, J., Zhu, X., Zhang, C., 2016. Homophily, structure, and content augmented network representation learning, in: Data Mining (ICDM), 2016 IEEE 16th International Conference on, pp. 609-618. 
[52] Zhao, Y., Levina, E., Zhu, J., et al., 2012. Consistency of community detection in networks under degree-corrected stochastic block models. The Annals of Statistics 40, 2266-2292. 